\title{
ALMOST SURE INVARIANCE PRINCIPLE FOR NON-AUTONOMOUS HOLOMORPHIC DYNAMICS IN $\mathbb{P}^{k}$
}

\author{
TURGAY BAYRAKTAR
}

Abstract. We prove an almost sure invariance principle, a strong form of approximation by Brownian motion, for non-autonomous holomorphic dynamical systems on complex projective space $\mathbb{P}^{k}$ for Hölder continuous and DSH observables.

\section{INTRODUCTION}

Let $f: \mathbb{P}^{k} \rightarrow \mathbb{P}^{k}$ be a holomorphic map of algebraic degree $d \geq 2$ and $\omega_{F S}$ denote the Fubini-Study form on $\mathbb{P}^{k}$ normalized by $\int \omega_{F S}^{k}=1$. Dynamical Green current $T_{f}$ of $f$ is defined to be the weak limit of the sequence of smooth forms $\left\{d^{-n}\left(f^{n}\right)^{*} \omega_{F S}\right\}$ (Bro65, HP94, FS95). Green currents play an important role in the dynamical study of holomorphic endomorphisms of the projective space [FS95, Sib99]. The current $T_{f}$ has Hölder continuous quasi-potentials, hence by Bedford-Taylor theory the exterior products

$$
T_{f}^{p}=T_{f} \wedge \cdots \wedge T_{f}
$$

are also well-defined for $1 \leq p \leq k$ and dynamically interesting currents. In particular, the top degree intersection $\mu_{f}=T_{f}^{k}$ yields the unique $f$-invariant measure of maximal entropy ([Lju83, BD01] ) with many interesting stochastic properties. For instance, in Dup10 Dupont obtained an almost sure invariance principle (ASIP) for the holomorphic dynamical system $\left(\mathbb{P}^{k}, \mathscr{B}, f, \mu_{f}\right)$ by using coding techniques and applying Philipp-Stout's theorem PS75] for observables with analytic singularities. The coding techniques were originally introduced by Przytycki-UrbańskiZdunik [PUZ89] in complex dimension one from which they deduced ASIP (see also Hay99 PRL07] and the references therein for some statistical results in the case of dimension one). Recall that ASIP in the context of a holomorphic dynamical system indicates that for a suitable class of observables $\psi$, the partial Birkhoff sums $\frac{1}{n} \sum_{j=0}^{n-1} \psi \circ f^{n}$ can be approximated by a Brownian motion (at integer times) in such a way that almost surely the error between the trajectories is negligible relative to the size of the trajectories (see $\$ 3$ for details). Some important statistical laws such as Central Limit Theorem (CLT) and Law of Iterated Logarithm (LIL) are among the immediate consequences of ASIP. In this context, CLT was also obtained by CLB05, DS06a, DNS10, by means of different methods. The approach of DinhNguyen-Sibony DNS10] is based on Gordin's martingale approximation method Gor69 and exponential decay of correlations for the Hölder continuous and DSH

Received by the editors April 8, 2017, and, in revised form, November 14, 2017, and January $14,2018$.

2010 Mathematics Subject Classification. Primary 37F10, 60F17, 32H50.

Key words and phrases. Almost sure invariance principle, Central Limit Theorem, holomorphic maps, non-autonomous dynamical systems. 
observables. Recall that a DSH function can be locally written as a difference of two plurisubharmonic (psh) functions.

In Bay15, we studied ergodic and statistical properties of random dynamical systems of holomorphic endomorphisms of $\mathbb{P}^{k}$. The latter is defined as successive iterations of holomorphic maps which are chosen at random according to a fixed probability measure. Under a mild assumption on the probability law, there is a naturally associated ergodic skew-product and a stationary homogenous Markov chain in that dynamical setting for which we established two versions of CLT. The first one is for the partial Birkhoff sums of the skew-product for Hölder continuous and DSH observables. The second one is for the backward images of randomly chosen points with respect to the stationary probability law of the Markov chain.

In the present paper, we consider non-autonomous holomorphic dynamical systems of the complex projective space $\mathbb{P}^{k}$. The latter is defined as successive iterations of a sequence of non-linear holomorphic maps of the same algebraic degree. In this setting, we obtain more refined statistical results. Namely, we prove an ASIP (Theorem 1.2) for these non-stationary systems under natural assumptions (1.1) and (1.2) on the distance of the tail of the sequence from the complement of holomorphic maps. It should be emphasized that the martingale approximation method of Gordin Gor69 gives rise to a reverse martingale increment sequence. Then using the martingale CLT, one can deduce CLT for the observations of a stationary dynamical system. The ASIP can often also be obtained in this way for the class of systems which are closed under time-reversal (see [MN05] and the references therein). However, for classes of non-stationary systems that are intrinsically time-orientated, such as non-autonomous holomorphic dynamics considered in this paper, the situation can be more delicate (see, e.g., MN05, CM15, HNTV17, KKM] for examples of real dynamical systems). Here, we utilize exponential decay of correlations for DSH and Hölder continuous observables and the abstract invariance principle obtained by CM15] in order to prove ASIP in the present setting.

1.1. Main result. Recall that the set of holomorphic endomorphisms $\mathcal{H}_{d}$ of fixed algebraic degree $d$ is a Zariski open subset of the set of meromorphic maps $\mathcal{M}_{d}$ and the complement $\mathcal{M}:=\mathcal{M}_{d} \backslash \mathcal{H}_{d}$ is an irreducible hypersurface GKZ94. The set $\mathcal{M}_{d}$ can be identified with $\mathbb{P}^{N}$ where $N=(k+1)\left(\begin{array}{c}d+k \\ d\end{array}\right)-1$. In what follows, we let $\operatorname{dist}(\cdot, \cdot)$ denote the distance induced by the Fubini-Study metric which is normalized here such that $\operatorname{dist}(\cdot, \cdot) \leq 1$.

For a sequence $\mathbf{f}:=\left(f_{0}, f_{1}, \ldots\right)$ of holomorphic maps $f_{n}: \mathbb{P}^{k} \rightarrow \mathbb{P}^{k}$ of fixed algebraic degree $d \geq 2$ we study statistical properties of the iterates

$$
F_{n}:=f_{n-1} \circ \cdots \circ f_{1} \circ f_{0}: \mathbb{P}^{k} \rightarrow \mathbb{P}^{k} .
$$

In Bay15 (see also DT13]), for such a sequence $\mathbf{f}=\left(f_{j}\right) \subset \mathcal{H}_{d}$ whose "tail" is sufficiently far from the complement $\mathcal{M}$, we proved the existence of a measure $\mu_{\mathbf{f}}$ which describes asymptotic distribution of pre-images of a generic point $z \in \mathbb{P}^{k}$.

Theorem 1.1 (Bay15). Let $\boldsymbol{f}=\left(f_{j}\right)_{j \geq 0} \subset \mathcal{H}_{d}$ be a sequence of holomorphic maps verifying

$$
\liminf _{n \rightarrow \infty} \frac{1}{n} \sum_{j=0}^{n-1} \log \operatorname{dist}\left(f_{j}, \mathcal{M}\right)>-\infty
$$


and

$$
\liminf _{n \rightarrow \infty} \frac{1}{j} \log \operatorname{dist}\left(f_{j}, \mathcal{M}\right)=0 .
$$

Then there exists a probability measure $\mu_{f}$ such that for every smooth probability measure $\nu$ on $\mathbb{P}^{k}$

$$
\frac{1}{d^{n k}} F_{n}^{*} \nu \rightarrow \mu_{f}
$$

in the sense of measures as $n \rightarrow \infty$. Moreover, the measure $\mu_{f}$ has Hölder continuous potentials.

We remark that since $\operatorname{dist}(\cdot, \cdot) \leq 1$ condition (1.2) is equivalent to

$$
\lim _{j \rightarrow \infty} \frac{1}{j} \log \operatorname{dist}\left(f_{j}, \mathcal{M}\right)=0 .
$$

Note that any bounded sequence (i.e., away from $\mathcal{M}$ ) falls in the framework of Theorem 1.1.

In \2, we provide an alternative proof for Hölder continuity of local potentials of invariant measures $\mu_{\mathbf{f}}$ given by Theorem [1.1. Furthermore, in Theorem 2.7 we obtain strong mixing properties of sequential holomorphic dynamical system $\left(\mathbb{P}^{k}, \mathscr{B}, \mathbf{f}, \mu_{\mathbf{f}}\right)$. As a consequence, we prove that the system $\left(\mathbb{P}^{k}, \mathscr{B}, \mathbf{f}, \mu_{\mathbf{f}}\right)$ is exact, a strong form of ergodicity (Theorem 2.5).

In what follows for a given sequence $\mathbf{f}=\left(f_{j}\right)_{j \geq 0} \subset \mathcal{H}_{d}$ we let $\mathbf{f}_{n}:=\left(f_{n}, f_{n+1}, \ldots\right)$ and denote the associated measure defined in Theorem 1.1 by $\mu_{n}$. Our main result gives an almost sure invariance principle (ASIP) for sequences of holomorphic endomorphisms verifying hypotheses (1.1) and (1.2) and for sequences of dsh and Hölder continuous observables.

Theorem 1.2 (ASIP). Let $\boldsymbol{f}=\left(f_{0}, f_{1}, \ldots\right)$ be a sequence of holomorphic maps of fixed algebraic degree $d \geq 2$ verifying (1.1) and (1.2). Let also $\psi_{n}$ be a sequence of $D S H$ (respectively, $\alpha$-Hölder continuous) functions satisfying $\sup _{n}\left\|\psi_{n}\right\|_{D S H}<\infty$ (respectively, $\left.\sup _{n}\left\|\psi_{n}\right\|_{\mathscr{C}_{\alpha}}<\infty\right)$. Assume that $\int_{\mathbb{P}^{k}} \psi_{n} d \mu_{n}=0$ and the variances $\sigma_{n}^{2}:=\int_{\mathbb{P}^{k}}\left(\sum_{j=0}^{n-1} \psi_{j} \circ F_{j}\right)^{2} d \mu_{f}$ satisfy

$$
C n^{\frac{1}{4}+\epsilon} \leq \sigma_{n} \text { for some } C, \epsilon>0 .
$$

Then on an extended probability space there exists a sequence of centered independent Gaussian random variables $\left(Z_{j}\right)_{j \in \mathbb{N}}$ such that $\sum_{j=0}^{\infty} \mathbb{E}\left[Z_{j}^{2}\right]=\sigma_{n}^{2}(1+o(1))$ and that

$$
\left|\sum_{j=0}^{n-1} \psi_{j} \circ F_{j}-\sum_{j=0}^{n-1} Z_{j}\right|=o\left(\sigma_{n}^{\gamma}\right) \quad \text { almost surely, }
$$

where $\frac{1+2 \epsilon}{1+4 \epsilon}<\gamma<1$.

Some remarks are in order. The assumption $\int_{\mathbb{p} k} \psi_{n} d \mu_{n}=0$ is not restrictive as we may replace $\psi_{n}$ with $\psi_{n}-\int_{\mathbb{P} k} \psi_{n} d \mu_{n}$. An immediate consequence of Theorem 1.2 is CLT, that is,

$$
\frac{1}{\sigma_{n}} \sum_{j=0}^{n-1} \psi_{j} \circ F_{j} \rightarrow \mathcal{N}(0,1)
$$


in distribution $\mu_{\mathbf{f}}$ (cf. [PS75, §1]). Another consequence is LIL which implies that

$$
\limsup _{n \rightarrow \infty}\left[\frac{1}{\sigma_{n} \sqrt{2 \log \log \left(\sigma_{n}\right)}} \sum_{j=0}^{n-1} \psi_{j} \circ F_{j}\right]=1 \text { almost surely. }
$$

Finally, we remark that for an autonomous holomorphic dynamical system (i.e., $f=f_{j} \forall j$ ) and a single observable (i.e., $\psi=\psi_{j} \forall j$ ) we have either $\sigma_{n}$ is bounded (in this case $\psi$ is a coboundary, i.e., $\psi=\zeta-\zeta \circ f$ for some $\zeta \in L_{\mu_{f}}^{2}$ ) or the $\sigma_{n}$ is of order $\sqrt{n}$. In this case, Theorem 1.2 implies that

$$
\left|\sum_{j=0}^{n-1} \psi \circ f^{j}-\sum_{j=0}^{n-1} Z_{j}\right|=o\left(n^{\gamma}\right) \text { almost surely, }
$$

where $\frac{3}{8}<\gamma<\frac{1}{2}$. Hence, we obtain a slightly improved version of Dup10, Theorem $\mathrm{C}]$ as Dupont's result does not give a lower bound for the exponent $\gamma$.

\section{ERGODICITY AND MIXING FOR NON-AUTONOMOUS SYSTEMS}

2.1. Invariant measures. For a sequence of endomorphisms $\mathbf{f}=\left(f_{j}\right)_{j \geq 0} \subset \mathcal{H}_{d}$ we define topological Lyapunov exponent

$$
\chi_{\text {top }}(\mathbf{f}):=\lim _{n \rightarrow \infty} \frac{1}{n} \log \sup _{x \in \mathbb{P} k}\left\|D_{x} F_{j}\right\|,
$$

where $\|\cdot\|$ is a fixed norm. Clearly, this definition does not depend on the choice of the norm. Note that the limit exists (possibly infinite) due to the sub-multiplicity of the sequence $\sup _{x \in \mathbb{P}^{k}}\left\|D_{x} F_{j}\right\|$. The next lemma will be useful in what follows.

Lemma 2.1. Let $\boldsymbol{f}=\left(f_{j}\right)_{j \geq 0} \subset \mathcal{H}_{d}$ verifying (1.1). Then $0<\chi_{\text {top }}(\boldsymbol{f})<\infty$.

Proof. Note that

$$
\begin{gathered}
\Psi: \mathcal{M}_{d} \times \mathbb{P}^{k} \rightarrow \mathbb{P}^{k} \\
\Psi(g, x)=g(x)
\end{gathered}
$$

defines a meromorphic map. Then it follows from [DD04, Lemma 2.1] that there exists $C>0$ and $q \geq 1$ such that

$$
\left\|D_{x} f_{j}\right\| \leq\left\|D_{f_{j}, x} \Psi\right\| \leq \operatorname{Cdist}\left(f_{j}, \mathcal{M}\right)^{-q} \forall j \geq 0 .
$$

Then by chain rule

$$
M_{n}:=\sup _{x \in \mathbb{P}^{k}}\left\|D_{x} F_{n}\right\| \leq C^{n} \prod_{j=0}^{n-1} \operatorname{dist}\left(f_{j}, \mathcal{M}\right)^{-q}
$$

and this in turn implies that

$$
\frac{1}{n} \log \left(\sup _{x \in \mathbb{P}^{k}}\left\|D_{x} F_{n}\right\|\right) \leq C-\frac{q}{n} \sum_{j=0}^{n-1} \log \operatorname{dist}\left(f_{j}, \mathcal{M}\right) .
$$

The following result was motivated by Sib99, Remark 1.7.2]. 
Theorem 2.2. Let $\boldsymbol{f}=\left(f_{j}\right)_{j \geq 0} \subset \mathcal{H}_{d}$ verifying (1.1) and (1.2). Then

$$
\frac{1}{d^{n}} F_{n}^{*} \omega_{F S} \rightarrow \omega_{F S}+d d^{c} g_{f}
$$

in the sense of currents. Moreover, Green function $g_{f}$ is $\alpha$-Hölder continuous for every $\alpha<\frac{\log d}{\chi_{t o p}(f)}$.

Proof. First, we sketch the proof of existence of the limit. Let $u_{j}$ be a smooth (quasi-plurisubharmonic) function defined by

$$
\frac{1}{d} f_{j}^{*} \omega_{F S}=\omega_{F S}+d d^{c} u_{j}
$$

Then

$$
\begin{aligned}
\frac{1}{d^{n}} F_{n}^{*} \omega_{F S} & =\frac{1}{d^{n}} f_{0}^{*} \cdots f_{n-1}^{*} \omega_{F S} \\
& =\omega_{F S}+d d^{c} \sum_{j=0}^{n-1} \frac{1}{d^{j}} u_{j} \circ F_{j} \\
& =: \quad \omega_{F S}+d d^{c} g_{n} .
\end{aligned}
$$

By [DT13, Proposition 3] there exists $C>0$ and $q \geq 1$ such that

$$
\left\|u_{j}\right\|_{\mathcal{C}^{1}} \leq \operatorname{Cdist}\left(f_{j}, \mathcal{M}\right)^{-q} \forall j \in \mathbb{N} .
$$

By (1.2) for each $\epsilon>0$ there exists $j_{0} \in \mathbb{N}$ such that

$$
\operatorname{dist}\left(f_{j}, \mathcal{M}\right) \geq e^{-\epsilon j} \forall j \geq j_{0} .
$$

Now,

$$
\begin{aligned}
\sup _{x \in \mathbb{P}^{k}}\left|g_{n}(x)-g_{m}(x)\right| & =\left|\sum_{j=n}^{m-1} \frac{1}{d^{j}} u_{j} \circ F_{j}\right| \\
& \leq \sum_{n+1}^{m-1} \frac{\sup _{x \in \mathbb{P}^{k}}\left|u_{j}(x)\right|}{d^{j}} \\
& \leq C \sum_{j=n}^{m-1} \frac{e^{\epsilon q j}}{d^{j}} .
\end{aligned}
$$

This imples that $g_{n} \rightarrow g_{\mathbf{f}}$ uniformly on $\mathbb{P}^{k}$ for some continuous qpsh function $g_{\mathbf{f}}$. In order to prove Hölder continuity, let $0<\alpha<\frac{\log d}{\chi_{t o p}(\mathbf{f})}$. Note that by (2.1)

$$
\operatorname{dist}\left(F_{n}(x), F_{n}(y)\right) \leq C^{n} M_{n} \operatorname{dist}(x, y) .
$$

Then by (2.3) and (2.4) for small $\epsilon>0$ we obtain

$$
\begin{aligned}
\left|g_{\mathbf{f}}(x)-g_{\mathbf{f}}(y)\right| & \leq \sum_{j \geq 0} \frac{\left|u_{j}\left(F_{j}(x)\right)-u_{j}\left(F_{j}(y)\right)\right|}{d^{j}} \\
& \leq \sum_{j \geq 0} \frac{C \operatorname{dist}\left(f_{j}, \mathcal{M}\right)^{-q} \operatorname{dist}\left(F_{j}(x), F_{j}(y)\right)^{\alpha}}{d^{j}} \\
& \leq \operatorname{Cdist}(x, y)^{\alpha} \sum_{j \geq 0} \frac{\operatorname{dist}\left(f_{j}, \mathcal{M}\right)^{-q} C^{\alpha j} e^{\chi_{t o p}(\mathbf{f}) \alpha j}}{d^{j}} \\
& \leq C_{1} \operatorname{dist}(x, y)^{\alpha} .
\end{aligned}
$$


Since the dynamical Green current $T_{\mathrm{f}}$ given by Theorem 2.2 has Hölder continuous potentials by Bedford-Taylor theory the exterior powers

$$
T_{\mathbf{f}}^{p}:=T_{\mathbf{f}} \wedge \cdots \wedge T_{\mathbf{f}}
$$

are also well-defined positive closed $(p, p)$ currents for each $1 \leq p \leq k$. Letting $T_{\mathbf{f}_{n}}$ be the dynamical Green current associated with $\mathbf{f}_{n}:=\left(f_{n}, f_{n+1}, \ldots\right)$ by (2.2) it is easy to see that they inherit the invariance properties

$$
f_{n-1}^{*} T_{\mathbf{f}_{n}}=d T_{\mathbf{f}_{n-1}} \text { and }\left(f_{n-1}\right)_{*} T_{\mathbf{f}_{n-1}}=d^{k-1} T_{\mathbf{f}_{n}} \quad \forall n \geq 1 .
$$

The next result is a direct consequence of [DT13, Proposition 11] and Theorem 1.1]

Proposition 2.3. The top degree dynamical Green current

$$
T_{f}^{k}=T_{f} \wedge \cdots \wedge T_{f}
$$

coincides with the measure $\mu_{f}$ given by Theorem 1.1. In particular, $\mu_{f}$ has continuous Hölder potentials and

$$
f_{n-1}^{*} \mu_{n}=d^{k} \mu_{n-1} \text { and }\left(f_{n-1}\right)_{*} \mu_{n-1}=\mu_{n} \quad \forall n \geq 1 .
$$

2.2. Sequential ergodicity, mixing, and exactness. In what follows we let $\mathscr{B}$ denote the Borel algebra on $\mathbb{P}^{k}$ and let $\left(\mathbb{P}^{k}, \mathscr{B}, \mathbf{f}, \mu_{\mathbf{f}}\right)$ be a sequential holomorphic dynamical system, that is, $\mathbf{f}=\left(f_{0}, f_{1}, \ldots\right)$ is a sequence of holomorphic maps $f_{j} \in \mathcal{H}_{d}$ satisfying (1.1) and (1.2), and let $\mu_{\mathrm{f}}$ be the measure given by Theorem 1.1. We say that the system $\left(\mathbb{P}^{k}, \mathscr{B}, \mathbf{f}, \mu_{\mathbf{f}}\right)$ is mean ergodic if

$$
\lim _{n \rightarrow \infty} \frac{1}{n} \sum_{j=0}^{n-1}\left[\mu_{\mathbf{f}}\left(B \cap F_{j}^{-1}(A)\right)-\mu_{\mathbf{f}}(B) \mu_{j}(A)\right]=0
$$

for all $\mathscr{B}$-measurable sets $A, B$. Note that (2.7) is equivalent to

$$
\lim _{n \rightarrow \infty} \frac{1}{n} \sum_{j=0}^{n-1}\left[\int_{\mathbb{P}^{k}} \varphi \cdot \psi \circ F_{j} d \mu_{\mathbf{f}}-\int_{\mathbb{P}^{k}} \varphi d \mu_{\mathbf{f}} \int_{\mathbb{P}^{k}} \psi \mu_{j}\right]=0
$$

for all continuous (equivalently smooth, bounded, or $L_{\mu_{\mathrm{f}}}^{2}$ ) functions $\varphi, \psi$ on $\mathbb{P}^{k}$. Using the property $P_{j} 1=1$ and the argument in [CR07, Remark 1.3] we see that (2.7) is equivalent to convergence in $L^{q}$-norm for $1 \leq q<\infty$ :

$$
\lim _{n \rightarrow \infty}\left\|\frac{1}{n} \sum_{j=0}^{n-1} \psi \circ F_{j}-\int \psi d \mu_{j}\right\|_{L_{\mu_{\mathrm{f}}}^{q}} \rightarrow 0 .
$$

Finally, we remark that in the setting of autonomous dynamical systems the mean ergodicity is equivalent to Birkhoff's ergodic theorem to which we refer here as pointwise ergodic. However, for non-autonomous systems these two concepts are not equivalent (cf. [CR07]). We say that the sequential holomorphic dynamical system $\left(\mathbb{P}^{k}, \mathscr{B}, \mathbf{f}, \mu_{\mathbf{f}}\right)$ is pointwise ergodic if

$$
\lim _{n \rightarrow \infty} \frac{1}{n} \sum_{j=0}^{n-1}\left[\psi \circ F_{j}(x)-\int \psi d \mu_{j}\right]=0 \quad \mu_{\mathbf{f}}-\text { a.s. }
$$

for all $L_{\mu_{\mathrm{f}}}^{2}$ functions $\psi$ on $\mathbb{P}^{k}$. 
We say that the system $\left(\mathbb{P}^{k}, \mathscr{B}, \mathbf{f}, \mu_{\mathbf{f}}\right)$ is mixing if for all smooth functions $\varphi, \psi$ on $\mathbb{P}^{k}$ we have

$$
\int_{\mathbb{P} k} \varphi \cdot \psi \circ F_{n} d \mu_{\mathbf{f}}-\int \varphi d \mu_{\mathbf{f}} \int \psi d \mu_{n} \rightarrow 0
$$

In Theorem 2.7, we prove that every sequential holomorphic dynamical system $\left(\mathbb{P}^{k}, \mathscr{B}, \mathbf{f}, \mu_{\mathbf{f}}\right)$ is mixing and hence mean ergodic. In fact, we obtain a stronger form of mean ergodicity.

For a given sequence $\mathbf{f}=\left(f_{j}\right)_{j \geq 0}$ of holomorphic maps $f_{j} \in \mathcal{H}_{d}$ we define

$$
\mathscr{B}_{j}:=F_{j}^{-1}(\mathscr{B})=f_{0}^{-1} \cdots f_{j-1}^{-1}(\mathscr{B}) .
$$

Note that

$$
\mathscr{B}=\mathscr{B}_{0} \supset \mathscr{B}_{1} \supset \cdots \supset \mathscr{B}_{n} .
$$

We also denote the asymptotic $\sigma$-algebra

$$
\mathscr{B}_{\infty}:=\bigcap_{j=0}^{\infty} \mathscr{B}_{j} .
$$

Definition 2.4. A sequential holomorphic dynamical system $\left(\mathbb{P}^{k}, \mathscr{B}, \mathbf{f}, \mu_{\mathbf{f}}\right)$ satisfying (1.1) and (1.2) is called exact if the asymptotic $\sigma$-algebra

$$
\mathscr{B}_{\infty}=\left\{\emptyset, \mathbb{P}^{k}\right\}
$$

modulo sets of $\mu_{\mathbf{f}}$-measure zero.

It follows from the definition of exact sequence that $\mathbf{f}$ is exact if and only if

$$
\bigcap_{j=0}^{\infty} L_{\mu_{\mathbf{f}}}^{2}\left(\mathscr{B}_{j}\right)=\{\text { constant functions }\},
$$

where

$$
L_{\mu_{\mathbf{f}}}^{2}\left(\mathscr{B}_{j}\right)=\left\{\xi \in L_{\mu_{\mathbf{f}}}^{2}\left(\mathbb{P}^{k}\right): \xi \text { is } \mathscr{B}_{j} \text { measurable }\right\} .
$$

In what follows we let $\mathbf{f}=\left(f_{j}\right)_{j \geq 0} \subset \mathcal{H}_{d}$ satisfying (1.1) and (1.2). Then each $f_{j} \in \mathcal{H}_{d}$ induces a unitary operator

$$
\begin{gathered}
T_{j}: L_{\mu_{j+1}}^{2}\left(\mathbb{P}^{k}\right) \rightarrow L_{\mu_{j}}^{2}\left(\mathbb{P}^{k}\right) \\
T_{j}(\varphi)=\varphi \circ f_{j} .
\end{gathered}
$$

We denote the adjoint of this operator by

$$
\begin{aligned}
& P_{j}: L_{\mu_{j}}^{2}\left(\mathbb{P}^{k}\right) \rightarrow L_{\mu_{j+1}}^{2}\left(\mathbb{P}^{k}\right) \\
& P_{j} \psi(x)=d^{-k} \sum_{f_{j}(y)=x} \psi(y)
\end{aligned}
$$

and let

$$
\mathcal{P}_{j}=P_{j-1} \cdots P_{1} P_{0}
$$

Theorem 2.5. Let $\boldsymbol{f}=\left(f_{j}\right)_{j \geq 0}$ be a sequence of holomorphic maps in $\mathcal{H}_{d}$ satisfying (1.1) and (1.2). Then the system $\left(\mathbb{P}^{k}, \mathscr{B}, \boldsymbol{f}, \mu_{f}\right)$ is exact. 
We remark that Theorem 2.5]generalizes Pet05] in which exactness was obtained for bounded sequences $\mathbf{f}=\left(f_{j}\right)$, i.e.,

$$
\operatorname{dist}\left(f_{j}, \mathcal{M}\right) \geq C>0 \forall j \geq 0 .
$$

The proof given in Pet05] is based on showing that for each $F_{j}$ there are sufficiently many inverse branches on a disc away from the critical values for which the preimages have small diameter. The latter argument is originally due to Briend and Duval BD01]. This method breaks down in the present setting and we provide a different approach.

Proof. Note that another equivalent condition for exactness of the sequence $\mathbf{f}=$ $\left(f_{j}\right)_{j \geq 0}$ is

$$
\left\|\mathcal{P}_{j} \psi\right\|_{L_{\mu_{j}}^{1}} \rightarrow 0 \text { as } j \rightarrow \infty
$$

for every $\psi \in L_{\mu_{\mathbf{f}}}^{1}$ such that $\int_{\mathbb{P}^{k}} \psi d \mu_{\mathbf{f}}=0$. Indeed, given such $\psi$ it follows from Doob's martingale convergence theorem that the conditional expectations

$$
\mathbb{E}\left[\psi \mid \mathscr{B}_{j}\right] \rightarrow \mathbb{E}\left[\psi \mid \mathscr{B}_{\infty}\right] \text { in } L_{\mu_{\mathbf{f}}}^{1}
$$

as $j \rightarrow \infty$. Since $\mathbb{E}\left[\psi \mid \mathscr{B}_{j}\right]=\left(\mathcal{P}_{j} \psi\right) \circ F_{j}$ (cf. Bay15, §5]) by invariance properties (2.6) we have

and the claim follows.

$$
\left\|\mathbb{E}\left[\psi \mid \mathscr{B}_{j}\right]\right\|_{L_{\mu_{\mathrm{f}}}^{1}}=\left\|\mathcal{P}_{j} \psi\right\|_{L_{\mu_{j}}^{1}}
$$

Finally, as $P_{j} 1 \equiv 1 \forall j$, it is enough to verify the condition (2.13) for smooth functions and this follows from Lemma 2.10 below.

Clearly, exactness implies mixing hence, mean ergodicity (2.8). Indeed, let $\varphi, \psi$ be smooth functions. We may assume that $\int_{\mathbb{P} k} \psi d \mu_{\mathbf{f}}=0$. Then by (2.13)

$$
\left|\int_{\mathbb{P}^{k}} \psi \cdot \varphi \circ F_{n} d \mu_{\mathbf{f}}\right|=\left|\int_{\mathbb{P}^{k}} \mathcal{P}_{n}(\psi) \cdot \varphi d \mu_{n}\right| \leq C_{\varphi}\left\|\mathcal{P}_{n} \psi\right\|_{L_{\mu_{n}}^{1}} \rightarrow 0 .
$$

Sequential mixing. In this section we explore mixing properties of the sequential holomorphic dynamical system $\left(\mathbb{P}^{k}, \mathscr{B}, \mathbf{f}, \mu_{\mathbf{f}}\right)$. We start with some preliminaries.

2.2.1. DSH functions. Recall that a function $\varphi$ is called a quasi-plurisubharmonic (qpsh for short) if $\varphi$ can be locally written as the sum of a smooth function and a pluri-subharmonic (psh) function. In what follows we denote by $L^{1}\left(\mathbb{P}^{k}\right)$ where the norm is given by Fubini-Study volume form. We say that a function $\psi \in L^{1}\left(\mathbb{P}^{k}\right)$ is dsh if outside a pluripolar set $\psi=\varphi_{1}-\varphi_{2}$ where $\varphi_{i}$ are qpsh functions. This implies that

$$
d d^{c} \psi=T^{+}-T^{-}
$$

for some positive closed $(1,1)$ currents $T^{ \pm}$. Two dsh functions are identified if they coincide outside a pluripolar set; we denote the set of all dsh functions by $D S H\left(\mathbb{P}^{k}\right)$. Note that dsh functions are stable under pull-back and push-forward operators induced by meromorphic self-maps of $\mathbb{P}^{k}$ and have good compactness properties inherited from those of qpsh functions. Following DS06b one can define a norm on $D S H\left(\mathbb{P}^{k}\right)$ as follows:

$$
\|\psi\|_{D S H}:=\|\psi\|_{L^{1}\left(\mathbb{P}^{k}\right)}+\inf \left\|T^{ \pm}\right\|,
$$

where $d d^{c} \psi=T^{+}-T^{-}$and the infimum is taken over all such representations. In what follows, $\lesssim$ and $\gtrsim$ denote inequalities up to a multiplicative constant. We 
remark that the currents $T^{ \pm}$have the same mass $\left\|T^{ \pm}\right\|:=\int_{\mathbb{P}^{k}} T^{ \pm} \wedge \omega_{F S}$ as they are cohomologous and $\|\cdot\|_{D S H} \lesssim\|\cdot\|_{\mathscr{C}^{2}}$. Moreover, it follows from properties of psh functions that $\|\cdot\|_{L^{p}} \lesssim\|\cdot\|_{D S H}$ for $1 \leq p<\infty$.

If $\mu$ is a probability measure on $\mathbb{P}^{k}$ such that all qpsh functions are $\mu$-integrable, then one can define

$$
\|\psi\|_{D S H}^{\mu}:=|\langle\mu, \psi\rangle|+\inf \left\|T^{ \pm}\right\|
$$

where $T^{ \pm}$as above and $\langle\mu, \psi\rangle:=\int_{\mathbb{P}^{k}} \psi d \mu$.

Proposition 2.6 ([DS06b] $)$. Let $\psi \in D S H\left(\mathbb{P}^{k}\right)$; then there exists negative qpsh functions $\varphi_{1}, \varphi_{2}$ such that $\psi=\varphi_{1}-\varphi_{2}$ and $d d^{c} \varphi_{i} \geq-c\|\psi\|_{D S H} \omega_{F S}$ where $c>0$ independent of $\psi$ and $\varphi_{i}$. Moreover, $|\psi|$ is also a dsh function and $\||\psi|\|_{D S H} \leq$ $c\|\psi\|_{D S H}$.

Now, we turn our attention to strong mixing properties of sequential holomorphic dynamical systems.

Theorem 2.7. Let $\left(\mathbb{P}^{k}, \mathscr{B}, \boldsymbol{f}, \mu_{f}\right)$ be a sequential holomorphic dynamical system satisfying (1.1) and (1.2). Then for every $\varphi \in L_{\mu_{n}}^{p}\left(\mathbb{P}^{k}\right)$ and $\psi \in D S H\left(\mathbb{P}^{k}\right)$ we have

$$
\left|\left\langle\mu_{f},\left(\varphi \circ f_{n-1} \circ \cdots \circ f_{0}\right) \psi\right\rangle-\left\langle\mu_{n}, \varphi\right\rangle\left\langle\mu_{f}, \psi\right\rangle\right| \leq C d^{-n}\|\varphi\|_{L_{\mu_{n}}^{p}}\|\psi\|_{D S H}^{\mu_{f}},
$$

where $C>0$ depends only on $\boldsymbol{f}$ and $p>1$. Moreover, for each $0 \leq \alpha \leq 2$ there exists $C>0$ depending only on $\boldsymbol{f}$ such that

$$
\left|\left\langle\mu_{f},\left(\varphi \circ f_{n-1} \circ \cdots \circ f_{0}\right) \psi\right\rangle-\left\langle\mu_{n}, \varphi\right\rangle\left\langle\mu_{f}, \psi\right\rangle\right| \leq C d^{-\frac{\alpha n}{2}}\|\varphi\|_{L_{\mu_{n}}^{p}}\|\psi\|_{\mathscr{C}^{\alpha}}
$$

for each $\varphi \in L_{\mu_{n}}^{p}$ and $\psi$ of class $\mathscr{C}^{\alpha}$.

We need several preliminary lemmas to prove Theorem 2.7. In what follows, $C_{\mathbf{f}}$ denotes a constant which depends only on $\mathbf{f}$.

Lemma 2.8. There exists $C_{f}>0$ such that

$$
\|\psi\|_{D S H}^{\mu_{n}} \leq C_{f}\|\psi\|_{D S H}
$$

for every $n \in \mathbb{N}$ and $\psi \in D S H\left(\mathbb{P}^{k}\right)$.

Proof. Let $\psi \in D S H\left(\mathbb{P}^{k}\right)$; then by Proposition 2.6 there exists qpsh functions $\phi_{i}$ such that $\psi=\phi_{1}-\phi_{2}$ and $d d^{c} \phi_{i} \geq-c\|\psi\|_{D S H} \omega_{F S}$ where $c>0$ is independent of $\psi$ and $\phi_{i}$. Since the measures $\mu_{n}$ have Hölder continuous super potentials (cf. [Bay15, Theorem 1.1]) with Hölder exponent $0<\alpha \leq 1$, by DN14, Lemma 3.3] we obtain

$$
\left|\left\langle\mu_{n}, \psi\right\rangle\right| \leq C_{\mathbf{f}} \max \left(\|\psi\|_{L^{1}\left(\mathbb{P}^{k}\right)}, c^{1-\alpha}\|\psi\|_{D S H}^{1-\alpha}\|\psi\|_{L^{1}}^{\alpha}\right) .
$$

If $\|\psi\|_{L^{1}\left(\mathbb{P}^{k}\right)} \geq c^{1-\alpha}\|\psi\|_{D S H}^{1-\alpha}\|\psi\|_{L^{1}}^{\alpha}$ we are done. Otherwise $\|\psi\|_{L^{1}\left(\mathbb{P}^{k}\right)}<c\|\psi\|_{D S H}$ and this implies that

$$
\left|\left\langle\mu_{n}, \psi\right\rangle\right| \leq c C_{\mathbf{f}}\|\psi\|_{D S H} .
$$

Thus, the assertion follows from Proposition 2.6.

Remark 2.9. By using a similar argument and using Lemma 2.8 one can also show that there exists a constant $C_{\mathbf{f}}>0$ such that

$$
\|\psi\|_{D S H} \leq C_{\mathbf{f}}\|\psi\|_{D S H}^{\mu_{n}}
$$

for every $n \geq 0$ (cf. [DT13, Proposition 8]).

The following lemma is essentially due to DNS10, however, we need to make some modifications to adapt it in our setting. 
Lemma 2.10. Let $1 \leq q<\infty$ and $\psi \in D S H\left(\mathbb{P}^{k}\right)$ satisfying

$$
\left\langle\mu_{f}, \psi\right\rangle=0
$$

then

$$
\left\|\mathcal{P}_{n}(\psi)\right\|_{L_{\mu_{j}}^{q}} \leq q C_{f} d^{-n}\|\psi\|_{D S H}^{\mu_{f}} \forall j, n \geq 1,
$$

where $C_{\boldsymbol{f}}>0$ depends only on the sequence $\boldsymbol{f}=\left(f_{j}\right)_{j \geq 0}$.

Proof. Note that $f_{n-1}^{*} \mu_{n}=d^{k} \mu_{n-1}$ for $n \geq 1$. This implies that

$$
\left\langle\mu_{n}, P_{n-1}\left(P_{n-2} \circ \cdots \circ P_{0} \psi\right)\right\rangle=0 .
$$

Moreover,

$$
\left\|P_{n-1}\left(P_{n-2} \circ \cdots \circ P_{0} \psi\right)\right\|_{D S H}^{\mu_{n}} \leq d^{-1}\left\|P_{n-2} \circ \cdots \circ P_{0} \psi\right\|_{D S H}^{\mu_{n-1}} .
$$

Indeed, we may write

$$
d d^{c}\left(P_{n-2} \circ \cdots \circ P_{0} \psi\right)=R_{n-2}^{+}-R_{n-2}^{-},
$$

where $R_{n-2}^{ \pm}$are some positive closed $(1,1)$ currents. Then

$$
\left\|P_{n-1}\left(P_{n-2} \circ \cdots \circ P_{0} \psi\right)\right\|_{D S H}^{\mu_{n}} \leq\left\|d^{-k}\left(f_{n-1}\right)_{*}\left(R_{n-2}^{ \pm}\right)\right\|=d^{-1}\left\|R_{n-2}^{ \pm}\right\|,
$$

where the last equality follows from cohomological computation. Now by Proposition 2.6. Remark 2.9, Lemma 2.8, and (2.15) we obtain

$$
\begin{aligned}
\left\|\left|P_{n-1} \circ \cdots \circ P_{1} \circ P_{0}(\psi)\right|\right\|_{D S H} & \leq C\left\|P_{n-1} \circ \cdots \circ P_{1} \circ P_{0}(\psi)\right\|_{D S H} \\
& \leq C_{1}\left\|P_{n-1} \circ \cdots \circ P_{1} \circ P_{0}(\psi)\right\|_{D S H}^{\mu_{n}} \\
& \leq C_{2} d^{-n}\|\psi\|_{D S H}^{\mu_{\mathrm{f}}} \\
& \leq C_{3} d^{-n}\|\psi\|_{D S H},
\end{aligned}
$$

where $C_{3}>0$ depends on $\mathbf{f}$ but does not depend on $n$ nor $\psi$. Thus, by the above estimate and Lemma 2.8 it is enough to prove the case $\|\psi\|_{D S H}>0$. Since

$$
\frac{d^{n}}{\|\psi\|_{D S H}}\left|P_{n-1} \circ \cdots \circ P_{1} \circ P_{0}(\psi)\right|
$$

is a bounded sequence in $D S H\left(\mathbb{P}^{k}\right)$, by Theorem 1.1 and DNS10, Corollary 1.2] (see also DN14, Proposition 4.4]) there exists $\gamma>0$ and $C_{\mathbf{f}}>0$ independent of $\psi$ such that

$$
\left\langle\mu_{j}, \exp \left(\gamma \frac{d^{n}}{\|\psi\|_{D S H}}\left|P_{n-1} \circ \cdots \circ P_{1} \circ P_{0}(\psi)\right|\right)\right\rangle \leq C_{\mathbf{f}}
$$

for all $j, n \geq 1$. Finally, by using the inequality $\frac{x^{q}}{q !} \leq e^{x}$ for $x \geq 0$ we conclude that

$$
\left\|P_{n-1} \circ \cdots \circ P_{1} \circ P_{0}(\psi)\right\|_{L_{\mu_{j}}^{q}} \leq q^{q} C_{\mathbf{f}, \gamma} d^{-n}\|\psi\|_{D S H} .
$$

In the autonomous case, as a consequence of interpolation theory between the Banach spaces $\mathscr{C}^{0}$ and $\mathscr{C}^{2}$ Tri78]; it was observed in DNS10] that a holomorphic map $f \in \mathcal{H}_{d}$ posseses a strong mixing property for $\alpha$-Hölder continuous functions with $0<\alpha \leq 1$ (see DNS10, Proposition 3.5]). Adapting their argument to our setting, we obtain the succeeding lemma. We omit the proof as it is similar to the one given in Lemma 2.10 and to that of [DNS10, Proposition 3.5]. 
Lemma 2.11. Let $1 \leq q<\infty$ and $0<\alpha \leq 1$ be fixed. If $\psi: \mathbb{P}^{k} \rightarrow \mathbb{R}$ is an $\alpha$-Hölder continuous function satisfying $\left\langle\mu_{f}, \bar{\psi}\right\rangle=0$, then there exists a constant $C_{f, \alpha}>0$ independent of $\psi$ such that

$$
\left\|\mathcal{P}_{n}(\psi)\right\|_{L^{q}\left(\mu_{n}\right)} \leq C_{\boldsymbol{f}, \alpha} q^{\frac{\alpha}{2}} d^{-\frac{n \alpha}{2}}\|\psi\|_{\mathscr{C}^{\alpha}}
$$

for every $n \geq 1$.

Proof of Theorem 2.7. If $\psi$ is constant, then the assertion follows from the invariance properties

$$
\left(f_{j}\right)_{*} \mu_{j}=\mu_{j+1} .
$$

Thus, replacing $\psi$ by $\psi-\left\langle\mu_{\mathbf{f}}, \psi\right\rangle$ we may assume that $\left\langle\mu_{\mathbf{f}}, \psi\right\rangle=0$. Then by Hölder's inequality and applying Lemma 2.10 with $q=\frac{p}{p-1}$ we obtain

$$
\begin{aligned}
\left|\left\langle\mu_{\mathbf{f}},\left(\varphi \circ f_{n-1} \circ \cdots \circ f_{0}\right) \psi\right\rangle\right| & =d^{-k n}\left|\left\langle F_{n}^{*} \mu_{n},\left(\varphi \circ f_{n-1} \circ \cdots \circ f_{0}\right) \psi\right\rangle\right| \\
& \leq\left|\left\langle\mu_{n}, \varphi P_{n-1} \circ \cdots \circ P_{1} \circ P_{0}(\psi)\right\rangle\right| \\
& \leq\|\varphi\|_{L^{p}\left(\mu_{n}\right)}\left\|P_{n-1} \circ \cdots \circ P_{1} \circ P_{0}(\psi)\right\|_{L^{q}\left(\mu_{n}\right)} \\
& \leq \frac{p}{p-1} C_{\mathbf{f}} d^{-n}\|\varphi\|_{L^{p}\left(\mu_{n}\right)}\|\psi\|_{D S H}^{\mu_{\mathbf{f}}}
\end{aligned}
$$

for some $c>0$ independent of $\psi$ and for all $n \geq 0$.

Finally, repeating the above argument by using Lemma 2.11 the second assertion follows.

The following result follows from Theorem 2.7 its proof is based on induction and Hölder's inequality. As the proof is similar to that of [DNS10, Theorem 3.4] we omit it.

Corollary 2.12. Let $\boldsymbol{f}=\left(f_{j}\right)_{j \in \mathbb{N}}$ and let $\mu_{j}$ be as in Theorem 2.7 and let $r \geq 1$ be an integer. Further, we let $\psi_{j}$ be dsh functions satisfying $\sup _{0 \leq j \leq r}\left\|\psi_{j}\right\|_{D S H}<\infty$. Then

$$
\left|\left\langle\mu_{f}, \psi_{0}\left(\psi_{1} \circ F_{n_{1}}\right) \cdots\left(\psi_{r} \circ F_{n_{r}}\right)\right\rangle-\prod_{j=0}^{r}\left\langle\mu_{n_{j}}, \psi_{j}\right\rangle\right| \leq C_{f} d^{-n} \prod_{j=0}^{r}\left\|\psi_{j}\right\|_{D S H},
$$

where $0=n_{0} \leq n_{1} \leq \cdots \leq n_{r}$ and $n:=\min _{0 \leq j \leq r}\left(n_{j+1}-n_{j}\right)$.

Note that we may also obtain an analogue statement of Corollary 2.12 for Hölder continuous functions. Next, we obtain a strong law of large numbers (SLLN) for dsh and Hölder continuous observables. This result will be used to establish ASIP (Theorem 1.2).

Theorem 2.13. Let $\left\{\phi_{n}\right\}_{n \in \mathbb{N}}$ be a sequence of dsh (respectively, Hölder continuous functions with exponent $0<\alpha \leq 1$ ) such that $\sup _{n \in \mathbb{N}}\left\|\phi_{n}\right\|_{D S H}<\infty$ (respectively, $\left.\sup _{n \in \mathbb{N}}\left\|\phi_{n}\right\|_{\mathscr{C}^{\alpha}}<\infty\right)$ and $\left\langle\mu_{n}, \phi_{n}\right\rangle=0$ for $n \geq 0$. Then for each integer $r \geq 1$ and $\delta>0$ we have

$$
\lim _{n \rightarrow \infty} \frac{1}{\sqrt{n}(\log n)^{2+\delta}} \sum_{j=0}^{n-1}\left(\phi_{j}^{r} \circ F_{j}-\left\langle\phi_{j}^{r}, \mu_{j}\right\rangle\right)=0 \mu_{f^{-}} \text {a.s. }
$$

Proof. We prove the theorem for dsh functions as the Hölder continuous case is similar. Letting

$$
X_{j}:=\phi_{j}^{r} \circ F_{j}-\left\langle\mu_{j}, \phi_{j}^{r}\right\rangle \text { for } j \geq 0
$$


by (2.6) we see that $\mathbb{E}\left[X_{j}\right]:=\int_{\mathbb{P}^{k}} X_{j} d \mu_{\mathbf{f}}=0$. Note that by Lemma 2.8 we have $\left\|\phi_{j}\right\|_{L_{\mu_{j}}^{p}} \lesssim\left\|\phi_{j}\right\|_{D S H}$ for $p \geq 1$ and we infer that

$$
\operatorname{Var}\left[X_{j}\right]:=\mathbb{E}\left[X_{j}^{2}\right]-\mathbb{E}\left[X_{j}\right]^{2}=\mathbb{E}\left[X_{j}^{2}\right]=O(1) .
$$

Recall that the covariance of $X_{j}$ and $X_{\ell}$ is given by

$$
\operatorname{Cov}\left(X_{j}, X_{\ell}\right):=\mathbb{E}\left[X_{j} X_{\ell}\right]-\mathbb{E}\left[X_{j}\right] \mathbb{E}\left[X_{\ell}\right]=\mathbb{E}\left[X_{j} X_{\ell}\right]
$$

Moreover, denoting $m_{j}:=\left\langle\mu_{j}, \phi_{j}\right\rangle$ by (2.6), triangle inequality, and Corollary 2.12 we have

$$
\begin{aligned}
\left|\operatorname{Cov}\left(X_{j}, X_{j+\ell}\right)\right| & \leq\left|\int_{\mathbb{P}^{k}} \phi_{j}^{r} \circ F_{j} \cdot \phi_{j+\ell}^{r} \circ F_{j+\ell} d \mu_{\mathbf{f}}\right|+\left|m_{j} m_{j+\ell}\right| \\
& \lesssim d^{-\ell}\left\|\phi_{j}\right\|_{D S H}^{r}\left\|\phi_{j+\ell}\right\|_{D S H}^{r}+d^{-2 j-\ell}\left\|\phi_{j}\right\|^{r}\left\|\phi_{j+\ell}\right\|^{r} \\
& \lesssim d^{-\ell}\left\|\phi_{j}\right\|_{D S H}^{r}\left\|\phi_{j+\ell}\right\|_{D S H}^{r}\left(1+d^{-2 j}\right)
\end{aligned}
$$

where the implied constant does not depend on $\ell$.

Hence, the assertion follows from Gal-Koksma SLLN [PS75, Theorem A1].

\section{ASIP}

First, we recall some basic notions that we will need in what follows. Let $\left(U_{j}\right)_{j \in \mathbb{N}}$ be a sequence of random variables on a probability space $(X, \mathscr{F}, \mu)$ such that $\mathbb{E}\left[U_{j}\right]=0 \forall j$. We say that $\left(U_{j}\right)_{j \in \mathbb{N}}$ satisfies an almost sure invariance principle (ASIP) with rates if there exists a sequence of independent centered Gaussian random variables $\left(Z_{j}\right)_{j \in \mathbb{N}}$ such that on an extended probability space

$$
\sum_{j=0}^{n-1} U_{j}=\sum_{j=0}^{n-1} Z_{j}+o\left(\sigma_{n}^{\gamma}\right) \text { almost surely, }
$$

where $\gamma \in(0,1)$ a fixed constant and $\sigma_{n}^{2}=\sum_{j=0}^{n-1} \mathbb{E}\left[Z_{j}^{2}\right] \rightarrow \infty$.

Recall that a Brownian motion at integer times coincides with a sum of i.i.d. Gaussian variables, hence the above definition can also be formulated as an almost sure approximation by a Brownian motion, with error $o\left(\sigma_{n}^{\gamma}\right)$.

A sequence of random variables $\left(U_{j}\right)_{j \in \mathbb{N}}$ is called a reversed martingale difference if there exists a non-increasing sequence $\left(\mathscr{F}_{j}\right)_{j \in \mathbb{N}}$ of $\sigma$-algebras (i.e., $\mathscr{F}_{j+1} \subset \mathscr{F}_{j}$ ) such that

(1) $U_{j}$ is $\mathscr{F}_{j}$-measurable,

(2) $\mathbb{E}\left[U_{j} \mid \mathscr{F}_{j+1}\right]=0 \forall j \geq 0$.

The next lemma will be useful in the proof of Theorem 1.2 .

Lemma 3.1 (CM15, Lemma 4.2]). Let $\left(\zeta_{n}\right)_{n \in \mathbb{N}}$ be a sequence of reversed martingale differences in $L_{\mu}^{p}$ for some $1 \leq p \leq 2$ with respect to a non-increasing filtration $\left(\mathscr{F}_{j}\right)_{j \in \mathbb{N}}$. Assume that

$$
\sum_{n \geq 0} \mathbb{E}\left[\left|\zeta_{n}\right|^{p}\right]<\infty
$$

then $\sum_{n \geq 0} \zeta_{n}$ converges $\mu$-a.s. and in $L_{\mu}^{p}$.

We will use the following abstract ASIP for sequences of reversed martingale differences. 
Theorem 3.2 (CM15). Let $\left(U_{j}\right)_{j \in \mathbb{N}}$ be a sequence of square integrable random variables adapted to a non-increasing filtration $\left(\mathscr{F}_{j}\right)_{j \in \mathbb{N}}$. Assume that $\mathbb{E}\left[U_{j} \mid \mathscr{F}_{j+1}\right]=$ $0 \mu$-a.s and $\nu_{n}^{2}:=\sum_{j=0}^{n-1} \mathbb{E}\left[U_{j}^{2}\right] \rightarrow \infty$ and that $\sup _{j} \mathbb{E}\left[U_{j}^{2}\right]<\infty$. Let also $a_{n}$ be a non-decreasing sequence of positive real numbers such that

$$
\left(a_{n} / \nu_{n}^{2}\right)_{n \in \mathbb{N}} \text { is non-increasing and }\left(a_{n} / \nu_{n}\right)_{n \in \mathbb{N}} \text { is non-decreasing. }
$$

Assume further that

$$
\begin{aligned}
& \sum_{j=0}^{n-1}\left(\mathbb{E}\left[U_{j}^{2} \mid \mathscr{F}_{j+1}\right]-\mathbb{E}\left[U_{j}^{2}\right]\right)=o\left(a_{n}\right) \mu-\text { a.s. } \\
& \sum_{j=0}^{\infty} a_{n}^{-q} \mathbb{E}\left[\left|U_{j}\right|^{2 q}\right]<\infty \text { for some } 1 \leq q \leq 2 .
\end{aligned}
$$

Then on an extended probability space there exists a sequence $\left(Z_{j}\right)_{j \in \mathbb{N}}$ of independent centered Gaussian variables such that $\mathbb{E}\left[Z_{j}^{2}\right]=\mathbb{E}\left[U_{j}^{2}\right]$ and

$$
\sup _{0 \leq k \leq n-1}\left|\sum_{j=0}^{k} U_{j}-\sum_{j=0}^{k} Z_{j}\right|=o\left(\left[a_{n}\left(\left|\log \left(\frac{\nu_{n}^{2}}{a_{n}}\right)\right|+\log \log a_{n}\right)\right]^{\frac{1}{2}}\right) \text { almost surely. }
$$

Proof of Theorem 1.2. We prove the theorem for DSH observables as the Hölder continuous case is similar. Let $\left(\mathscr{B}_{j}\right)_{j \in \mathbb{N}}$ denote the filtration defined by (2.12). Note that

$$
\mathcal{P}_{n}\left(\psi_{n} \circ F_{n}\right)=\psi_{n} .
$$

Moreover, since $\mathbb{E}\left[\phi \mid \mathscr{B}_{n}\right]$ is an orthogonal projection of $\phi \in L^{2}\left(\mathscr{B}, \mu_{\mathbf{f}}\right)$ to the Hilbert space $L^{2}\left(\mathscr{B}_{n}, \mu_{\mathbf{f}}\right)$ it is easy to see that (cf. Bay15, §5])

$$
\mathbb{E}\left[\psi_{n} \circ F_{n} \mid \mathscr{B}_{n}\right]=\left(\mathcal{P}_{n} \psi_{n}\right) \circ F_{n} \mu_{\mathbf{f}}-\text { a.s. }
$$

This implies that

$$
\left\|\mathbb{E}\left[\psi_{n} \circ F_{n} \mid \mathscr{B}_{n}\right]\right\|_{L_{\mu_{\mathbf{f}}}^{2}}=\left\|\mathcal{P}_{n} \psi_{n}\right\|_{L_{\mu_{n}}^{2}} \leq C_{\mathbf{f}} d^{-n}\left\|\psi_{n}\right\|_{D S H}^{\mu_{\mathbf{f}}},
$$

where the latter inequality follows from Lemma 2.10.

Now, we define

$$
h_{j}=P_{j-1}\left(\psi_{j-1}+h_{j-1}\right) \forall j \geq 1
$$

and set $h_{0} \equiv 0$. We also let

$$
U_{j}:=\left(\psi_{j}+h_{j}-h_{j+1} \circ f_{j}\right) \circ F_{j} \forall j \geq 0 .
$$

Note that since $P_{j}\left(\psi_{j}+h_{j}\right)-h_{j+1}=0$, by (3.5) we obtain

$$
\mathbb{E}\left[U_{j} \mid \mathscr{B}_{j+1}\right]=0 .
$$

Hence, $\left(U_{j}\right)_{j \in \mathbb{N}}$ form a sequence of reversed martingale differences for the filtration $\left(\mathscr{B}_{j}\right)_{j \in \mathbb{N}}$ defined by (2.12). Moreover,

$$
\sum_{j=0}^{n-1} U_{j}=S_{n}-h_{n} \circ F_{n}
$$

where $S_{n}:=\sum_{j=0}^{n-1} \psi_{j} \circ F_{j}$. Furthermore, by invariance properties (2.6) and Lemma 2.10 we have

$$
\left\|h_{n} \circ F_{n}\right\|_{L_{\mu_{\mathrm{f}}}^{2}}=\left\|h_{n}\right\|_{L_{\mu_{n}}^{2}} \leq C_{1}
$$


where $C_{1}>0$ depends only on $\mathbf{f}$ but independent of $n$. This implies that

$$
\begin{aligned}
\left\|S_{n}\right\|_{L_{\mu_{\mathbf{f}}}^{2}}-\left\|\sum_{j=0}^{n-1} U_{j}\right\|_{L_{\mu_{\mathbf{f}}}^{2}} \mid & \leq\left\|S_{n}-\sum_{j=0}^{n-1} U_{j}\right\|_{L_{\mu_{\mathbf{f}}}^{2}} \\
& =\left\|h_{n} \circ F_{n}\right\|_{L_{\mu_{\mathbf{f}}}^{2}} \leq C_{1}
\end{aligned}
$$

and we conclude that $\left\|\sum_{j=0}^{n-1} U_{j}\right\|_{L_{\mu_{\mathbf{f}}}^{2}}^{2} \rightarrow \infty$. Then using $\int_{\mathbb{P}^{k}} U_{i} U_{j} d \mu_{\mathbf{f}}=0$ for $i \neq j$ we deduce that

$$
\nu_{n}^{2}=\sum_{j=0}^{n-1} \mathbb{E}\left[U_{j}^{2}\right]=\left\|\sum_{j=0}^{n-1} U_{j}\right\|_{L_{\mu_{\mathrm{f}}}^{2}}^{2} \rightarrow \infty,
$$

where $\mathbb{E}\left[U_{j}^{2}\right]=\int_{\mathbb{P}^{k}} U_{j}^{2} d \mu_{\mathbf{f}}$. Moreover, by (3.9)

$$
\left|\sigma_{n}-\nu_{n}\right| \leq C_{1}
$$

We also remark that by Lemma 2.10

$$
\sup _{j}\left\|U_{j}\right\|_{L_{\mu_{\mathrm{f}}}^{q}} \leq C_{q}, \quad \forall q \geq 1 .
$$

First, we establish ASIP for $\left(U_{j}\right)_{j \geq 0}$ by verifying the hypotheses (3.2) and (3.3) of Theorem 3.2. To this end let $\frac{1+\epsilon}{1+4 \epsilon}<\gamma<1$ and we choose $a_{n}=\nu_{n}^{2 \gamma} \gtrsim n^{\frac{1}{2}+\epsilon}$ which is clearly a non-decreasing sequence of positive real numbers verifying $a_{n} / \nu_{n}^{2}=\nu_{n}^{2 \gamma-2}$ is decreasing and $a_{n} / \nu_{n}=\nu_{n}^{2 \gamma-1} \uparrow \infty$ since $\gamma>\frac{1}{2}$. Note that by (3.11), (3.10), and the assumption (1.3) on $\sigma_{n}$ we obtain

$$
\sum_{j \geq 0} \frac{\left\|U_{j}\right\|_{L_{\mu_{\mathbf{f}}}^{4}}^{4}}{a_{j}^{2}} \leq \sum_{j \geq 1} \frac{C_{3}}{j^{1+4 \epsilon}}<\infty
$$

which verifies (3.3) with $q=2$. Moreover, by (3.12) and Lemma 3.1

$$
\sum_{j=0}^{\infty} \frac{\mathbb{E}\left[U_{j}^{2} \mid \mathscr{B}_{j+1}\right]-U_{j}^{2}}{a_{j}}
$$

converges $\mu_{\mathrm{f}}$-a.s. Then by Kronecker's Lemma Dur10, Theorem 2.5.5]

$$
\sum_{j=0}^{n-1}\left(\mathbb{E}\left[U_{j}^{2} \mid \mathscr{B}_{j+1}\right]-U_{j}^{2}\right)=o\left(a_{n}\right) \mu_{\mathbf{f}} \text {-a.s. }
$$

Hence, in order to get (3.3), it is enough to show that

$$
\sum_{j=0}^{n-1}\left(U_{j}^{2}-\mathbb{E}\left[U_{j}^{2}\right]\right)=o\left(a_{n}\right) \mu_{\mathbf{f}} \text {-a.s. }
$$

Let us denote by

$$
\phi_{j}:=\left(\psi_{j}+h_{j}-h_{j+1} \circ f_{j}\right)
$$

so that $U_{j}^{2}=\phi_{j}^{2} \circ F_{j}$ for $j \geq 0$. Note that $\phi_{j}$ are dsh functions and by Lemma 2.10 their dsh norms are bounded. Moreover, since $\left\langle\mu_{j}, h_{j}\right\rangle=0$ by (2.6) we have $\left\langle\mu_{j}, \phi_{j}\right\rangle=0$ for $j \geq 0$. Hence, the claim (3.13) follows from Theorem 2.13. 
Now, applying Theorem 3.2 for $\left(U_{j}\right)_{j \geq 0}$ on an extended probability space we obtain centered independent Gaussian random variables $\left(Z_{j}\right)_{j \geq 0}$ such that $\mathbb{E}\left[Z_{j}^{2}\right]=$ $\mathbb{E}\left[U_{j}^{2}\right]$ and

$$
\begin{aligned}
\sup _{0 \leq k \leq n-1}\left|\sum_{j=0}^{k} U_{j}-\sum_{j=0}^{k} Z_{j}\right| & =o\left(\sqrt{a_{n}\left(\left|\log \left(\frac{\nu_{n}^{2}}{a_{n}}\right)\right|+\log \log a_{n}\right)}\right) \\
& =o\left(\sigma_{n}^{\gamma} \sqrt{\log \sigma_{n}}\right) \text { almost surely, }
\end{aligned}
$$

where the last equality follows from (3.10).

Finally, we remark that $h_{j}$ are dsh functions and by Lemma 2.10 their dsh norms are bounded. Moreover, by (2.6) this means $\left\langle\mu_{j}, h_{j}\right\rangle=0$ and by Theorem 2.13

$$
\sum_{j=0}^{n-1} h_{j} \circ F_{j}=o\left(\sigma_{n}^{\gamma} \sqrt{\log \sigma_{n}}\right) \text { almost surely. }
$$

Since the log-term can be absorbed in $\sigma_{n}^{\gamma}$ when $\gamma$ varies in an open interval, (1.4) follows from (3.7), (3.8). This finishes the proof for DSH observables.

For the Hölder continuous observables, Theorem 2.13 does not apply directly to the functions $\phi_{j}$. In this case, using a standard convolution and a partition of unity, we can approximate $\psi_{j}$ by functions $\psi_{j, \delta}$ satisfying

$$
\left\|\psi_{j, \delta}\right\|_{\mathscr{C}^{2}} \lesssim \delta^{-2} \text { and } \sup _{\mathbb{P}^{k}}\left|\psi_{j}(x)-\psi_{j, \delta}(x)\right| \lesssim \delta^{r}
$$

for some $r>0$. Then we estimate the covariances as in Theorem 2.13 and obtain SLLN. The details are left to the reader.

\section{ACKNOWLEDGment}

I would like to thank the referee whose comments improved the presentation of this paper.

\section{REFERENCES}

[Bay15] Turgay Bayraktar, Ergodic properties of random holomorphic endomorphisms of $\mathbb{P}^{k}$, Int. Math. Res. Not. IMRN 4 (2015), 927-959, DOI 10.1093/imrn/rnt226. MR.3340342

[Bay15a] Turgay Bayraktar, Corrigendum to: Ergodic properties of random holomorphic endomorphisms of $\mathbb{P}^{k}$ [ MR3340342], Int. Math. Res. Not. IMRN (2015), no. 14, 6005-6009. MR.3384466

[BD01] Jean-Yves Briend and Julien Duval, Deux caractérisations de la mesure d'équilibre d'un endomorphisme de $\mathrm{P}^{k}(\mathbf{C})$ (French, with English and French summaries), Publ. Math. Inst. Hautes Études Sci. 93 (2001), 145-159, DOI 10.1007/s10240-001-8190-4. MR.1863737

[Bro65] Hans Brolin, Invariant sets under iteration of rational functions, Ark. Mat. 6 (1965), 103-144 (1965), DOI 10.1007/BF02591353. MR0194595

[CLB05] Serge Cantat and Stéphane Le Borgne, Théorème limite central pour les endomorphismes holomorphes et les correspondances modulaires (French), Int. Math. Res. Not. 56 (2005), 3479-3510, DOI 10.1155/IMRN.2005.3479. MR2200586

[CM15] Christophe Cuny and Florence Merlevède, Strong invariance principles with rate for "reverse" martingale differences and applications, J. Theoret. Probab. 28 (2015), no. 1, 137-183, DOI 10.1007/s10959-013-0506-z. MR.3320963

[CR07] Jean-Pierre Conze and Albert Raugi, Limit theorems for sequential expanding dynamical systems on [0,1], Ergodic theory and related fields, Contemp. Math., vol. 430, Amer. Math. Soc., Providence, RI, 2007, pp. 89-121, DOI 10.1090/conm/430/08253. MR 2331327 
[DD04] Tien-Cuong Dinh and Christophe Dupont, Dimension de la mesure d'équilibre d'applications méromorphes (French, with English summary), J. Geom. Anal. 14 (2004), no. 4, 613-627, DOI 10.1007/BF02922172. MR2111420

[DN14] Tien-Cuong Dinh and Viêt-Anh Nguyên, Characterization of Monge-Ampère measures with Hölder continuous potentials, J. Funct. Anal. 266 (2014), no. 1, 67-84, DOI 10.1016/j.jfa.2013.08.026. MR3121721

[DNS10] Tien-Cuong Dinh, Viêt-Anh Nguyên, and Nessim Sibony, Exponential estimates for plurisubharmonic functions and stochastic dynamics, J. Differential Geom. 84 (2010), no. 3, 465-488. MR2669362

[DS06a] Tien-Cuong Dinh and Nessim Sibony, Decay of correlations and the central limit theorem for meromorphic maps, Comm. Pure Appl. Math. 59 (2006), no. 5, 754-768, DOI 10.1002/cpa.20119. MR2172806

[DS06b] Tien-Cuong Dinh and Nessim Sibony, Distribution des valeurs de transformations méromorphes et applications (French, with English summary), Comment. Math. Helv. 81 (2006), no. 1, 221-258, DOI 10.4171/CMH/50. MR2208805

[DT13] Henry de Thélin, Endomorphismes pseudo-aléatoires dans les espaces projectifs I (French, with English and French summaries), Manuscripta Math. 142 (2013), no. 3-4, 347-367, DOI 10.1007/s00229-012-0603-9. MR.3117166

[Dup10] Christophe Dupont, Bernoulli coding map and almost sure invariance principle for endomorphisms of $\mathbb{P}^{k}$, Probab. Theory Related Fields 146 (2010), no. 3-4, 337-359, DOI 10.1007/s00440-008-0192-4. MR2574731

[Dur10] Rick Durrett, Probability: theory and examples, 4th ed., Cambridge Series in Statistical and Probabilistic Mathematics, vol. 31, Cambridge University Press, Cambridge, 2010. MR.2722836

[FS95] John Erik Fornaess and Nessim Sibony, Complex dynamics in higher dimension. II, Modern methods in complex analysis (Princeton, NJ, 1992), Ann. of Math. Stud., vol. 137, Princeton Univ. Press, Princeton, NJ, 1995, pp. 135-182. MR.1369137

[GKZ94] I. M. Gel'fand, M. M. Kapranov, and A. V. Zelevinsky, Discriminants, resultants, and multidimensional determinants, Mathematics: Theory \& Applications, Birkhäuser Boston, Inc., Boston, MA, 1994. MR 1264417

[Gor69] M. I. Gordin, The central limit theorem for stationary processes (Russian), Dokl. Akad. Nauk SSSR 188 (1969), 739-741. MR0251785

[Hay99] Nicolai Haydn, Convergence of the transfer operator for rational maps, Ergodic Theory Dynam. Systems 19 (1999), no. 3, 657-669, DOI 10.1017/S0143385799130190. MR.1695914

[HNTV17] Nicolai Haydn, Matthew Nicol, Andrew Török, and Sandro Vaienti, Almost sure invariance principle for sequential and non-stationary dynamical systems, Trans. Amer. Math. Soc. 369 (2017), no. 8, 5293-5316, DOI 10.1090/tran/6812. MR/3646763

[HP94] John H. Hubbard and Peter Papadopol, Superattractive fixed points in $\mathbf{C}^{n}$, Indiana Univ. Math. J. 43 (1994), no. 1, 321-365, DOI 10.1512/iumj.1994.43.43014. MR. 1275463

[Lju83] M. Ju. Ljubich, Entropy properties of rational endomorphisms of the Riemann sphere, Ergodic Theory Dynam. Systems 3 (1983), no. 3, 351-385, DOI 10.1017/S0143385700002030. MR741393

[KKM] A. Korepanov, Z. Kosloff, and I. Melbourne, Martingale-coboundary decomposition for families of dynamical systems, Ann. Inst. H. Poincaré Anal. Non Linéaire 35 (2018), no. 4, 859-885, DOI 10.1016/j.anihpc.2017.08.005. MR.3795019

[MN05] Ian Melbourne and Matthew Nicol, Almost sure invariance principle for nonuniformly hyperbolic systems, Comm. Math. Phys. 260 (2005), no. 1, 131-146, DOI 10.1007/s00220-005-1407-5. MR2175992

[Pet05] Han Peters, Non-autonomous dynamics in $\mathbb{P}^{k}$, Ergodic Theory Dynam. Systems 25 (2005), no. 4, 1295-1304, DOI 10.1017/S0143385704000987. MR2158406

[PRL07] Feliks Przytycki and Juan Rivera-Letelier, Statistical properties of topological ColletEckmann maps (English, with English and French summaries), Ann. Sci. École Norm. Sup. (4) 40 (2007), no. 1, 135-178, DOI 10.1016/j.ansens.2006.11.002. MR2332354

[PS75] Walter Philipp and William Stout, Almost sure invariance principles for partial sums of weakly dependent random variables, Mem. Amer. Math. Soc. 2 issue 2, 161 (1975), iv+140. MR 0433597 
[PUZ89] Feliks Przytycki, Mariusz Urbański, and Anna Zdunik, Harmonic, Gibbs and Hausdorff measures on repellers for holomorphic maps. I, Ann. of Math. (2) 130 (1989), no. 1, 1-40, DOI 10.2307/1971475. MR1005606

[Sib99] Nessim Sibony, Dynamique des applications rationnelles de $\mathbf{P}^{k}$ (French, with English and French summaries), Dynamique et géométrie complexes (Lyon, 1997), Panor. Synthèses, vol. 8, Soc. Math. France, Paris, 1999, pp. ix-x, xi-xii, 97-185. MR 1760844

[Tri78] Hans Triebel, Interpolation theory, function spaces, differential operators, NorthHolland Mathematical Library, vol. 18, North-Holland Publishing Co., AmsterdamNew York, 1978. MR503903

Faculty of Engineering and Natural Sciences, Sabanci University, İstanbul, Turkey

Email address: tbayraktar@sabanciuniv.edu 